\title{
Viewpoint
}

\section{Superconductivity and magnetism living apart together?}

\author{
Stefano Gariglio and Jean-Marc Triscone \\ DPMC-MaNEP, University of Geneva, 24 Quai Ernest-Ansermet, CH-1211 Geneva, Switzerland
}

\author{
Andrea Caviglia \\ Max Planck Research Department for Structural Dynamics, University of Hamburg-CFEL, Hamburg, \\ Germany
}

Published July 25, 2011

The interface between two insulators is found to display ferromagnetism and superconductivity.

Subject Areas: Strongly Correlated Materials

\author{
A Viewpoint on: \\ Coexistence of Superconductivity and Ferromagnetism in Two Dimensions \\ D. A. Dikin, M. Mehta, C. W. Bark, C. M. Folkman, C. B. Eom, and V. Chandrasekhar \\ Physical Review Letters 107, 056802 2011 - Published July 25, 2011
}

Strong electronic correlations in complex oxides lead to a wealth of electronic properties and rich phase diagrams. $\mathrm{SrTiO}_{3}$ (STO), a widely studied prototype perovskite compound that is insulating when undoped, displays an amazing variety of instabilities. These include a transition into a superconducting state upon charge doping or into ferroelectric order under pressure or strain.

Great excitement came from the discovery in 2004 of a two-dimensional electron gas (2DEG) at the interface between a (001) $\mathrm{TiO}_{2}$ terminated STO crystal and an epitaxial $\mathrm{LaAlO}_{3}$ (LAO) film [1] [see Fig. 11(a)]. In 2007, Reyren et al. discovered that the interface between these two band insulators is superconducting below $200 \mathrm{mK} 2$. Successive experiments have demonstrated that this twodimensional superconducting state can be switched on and off by applying an electric field 3. Now, writing in Physical Review Letters, Dmitriy Dikin and collaborators from Northwestern University and the University of Wisconsin, Madison, both in the US, describe a series of experiments revealing signs of magnetism "mixed" with superconducting behavior in the transport properties of LAO/STO interfaces [4. Magnetic hysteresis at the interface was previously reported by Brinkman and co-workers, however, this effect was observed in samples not displaying superconductivity [5].

Differences in the electronic properties of nominally identical interfaces can be traced back to the seminal paper of Ohtomo and Hwang [1]. During the LAO layer deposition onto a STO single crystal, the thermodynamic conditions (mainly temperature and oxygen pressure) used in the growth chamber affect the oxygen content of the STO substrate as well as the disorder in the interface region - two critical parameters. The observed sensitivity of the electronic properties to possible small changes in stoichiometry and disorder might be a signa-

DOI: $10.1103 /$ Physics.4.59

URL: http://link.aps.org/doi/10.1103/Physics .4.59 ture of the proximity in energy of different ground states. A key issue is, however, to understand if magnetism and superconductivity coexist on the same sample in different areas - the system displaying phase separation-or coexist in a single phase. A related question is whether the magnetic and superconducting properties originate from the same Ti3d electrons. Several recent reports suggest phase separation. Ariando et al. observed a coexistence of metallicity and magnetism [6], while Li et al., using high-resolution torque and magnetotransport, demonstrated that magnetism and superconductivity coexist on the same samples but most likely in different areas of the interface [7] (a finding in agreement with unpublished scanning SQUID investigations [8]). In a recent theoretical paper, Pavlenko et al. showed that oxygen vacancies could locally lead to an enhanced charge density and favor a ferromagnetic state, possibly explaining the source of the observed phase separation [9] [see Fig. 1 (c)]. In this scenario-pointing to the importance of the growth conditions - magnetism is induced by oxygen vacancies, whereas the metallic-superconducting behavior can be traced back to an intrinsic doping mechanism. Other electronic configurations can also lead to magnetism. Those require that the electrons sit alternatively on the Ti atoms of the interface layer, like black squares on a checkerboard [10]. It is worth noting here that the interface layer is equivalent to $\mathrm{LaTiO}_{3}$, a system that itself is an antiferromagnetic insulator due to strong electronic correlations. Such a magnetic structure requires a perfectly homogenous interface and that the electrons localize on one atomic layer. However, more recent $a b$ initio calculations, which take into account the particular dielectric properties of STO, provide a description where electrons delocalize over a few unit cells inside the substrate [11].

(c) 2011 American Physical Society 
(a)

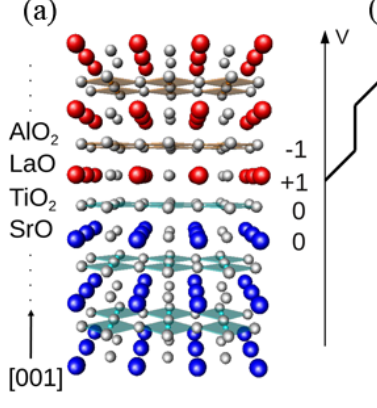

(c)

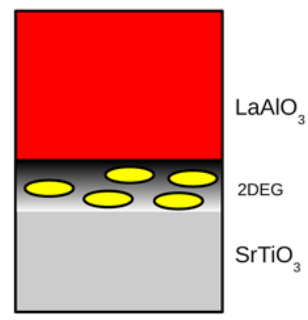

FIG. 1: (a) The $\mathrm{LaAlO}_{3} / \mathrm{SrTiO}_{3}$ interface. At the interface, a polar discontinuity between neutral $\mathrm{SrTiO}_{3}$ planes and charged $\mathrm{LaAlO}_{3}$ planes produces a diverging potential. For $\mathrm{LaAlO}_{3}$ thicknesses above three unit cells, an electronic reconstruction occurs, transferring from the $\mathrm{LaAlO}_{3}$ surface to the interface $1 / 2$ electron per unit cell 13]. (b) From Dikin et al. 4], hysteresis in the magnetic-field dependence of sample resistance for different temperatures at a fixed gate voltage, $V_{g}=-100 \mathrm{~V}$. (c) Schematic of the phase separation scenario. Pavlenko et al. 10 propose that ferromagnetism is linked to oxygen vacancies, which locally produce an increase in carrier density favoring localized ferromagnetic regions (yellow), and that the electronic reconstruction is responsible for the metallic and superconducting regions. (Credit: (a,c) S. Gariglio; (b) D. A. Dikin et al. 4])

The report of Dikin et al. features a ten-unit-cell LAO film grown by pulsed laser deposition at an oxygen pressure of $10^{-3}$ mbar, a pressure regime that in previous studies led to superconducting samples (notice, however, that no in situ oxygen annealing was used here). While it is clear that an oxygen-starved growth environment favors 3D metallicity at the interface because of the creation of a large amount of oxygen vacancies, the occurrence of ferromagnetism may be linked not only to the used oxygen growth pressure but also to inhomogeneities in the doping profile, in directions both normal and par- allel to the interface.

Analyzing the longitudinal and Hall resistances as a function of the gate voltage used to control the carrier density, Dikin et al. suggest that two independent electronic gases contribute to the conduction at the interface. The observed hysteretic behavior of the magnetoresistance, limited to very low temperatures in the superconducting region $\left[T_{c}(R=0)<T<T_{c}\right.$ onset] and slightly above [see Fig. 1(b)], may suggest that superconductivity and magnetism originate from the same phase. Superconductivity and ferromagnetism are, in general, exclusive phenomena. Superconducting ferromagnetic systems have, however, been found, albeit with a complex order parameter. A notable example is $\mathrm{UGe}_{2} 12$, where superconductivity arises from the same electrons responsible for ferromagnetism.

The variety of phenomena observed at the LAO/STO interface is very promising for the use and possible tuning and control of the interface properties. At the same time, several key, fascinating questions are open, from the detailed understanding of the parameters controlling the material ground state and possible phase separation to questions related to the nature of the superconducting state. Further detailed studies are necessary to understand whether this interfacial system has the key ingredients necessary to allow for a mixed ground state.

\section{References}

[1] A. Ohtomo and H. Y. Hwang, Nature 427, 423 (2004).

[2] N. Reyren et al., Science 317, 1197 (2007).

[3] A. D. Caviglia et al., Nature 456, 624 (2008).

[4] D. A. Dikin, M. Mehta, C. W. Bark, C. M. Folkman, C. B. Eom, and V. Chandrasekhar, Phys. Rev. Lett 107, 056802 (2011).

[5] A. Brinkman et al., Nature Mater. 6, 493 (2007).

[6] Ariando et al., Nature Commun. 2, 188 (2011).

[7] L. Li, C. Richter, J. Mannhart, and R. C. Ashoori, arXiv:1105.0235v1 (2011).

[8] K. Moler (private communications).

[9] N. Pavlenko, T. Kopp, E.Y. Tsymbal, G. A. Sawatzky, and J. Mannhart, arXiv:1105.1163v1 (2011).

[10] R. Pentcheva and W. E. Pickett, Phys. Rev. B 74, 35112 (2006).

[11] See, for instance, P. Delugas, et al., Phys. Rev. Lett. 106, 166807 (2011).

[12] S. S. Saxena et al., Nature 406, 587 (2000).

[13] S. Thiel et al., Science 313, 1942 (2006). 


\section{About the Authors}

\section{Stefano Gariglio}

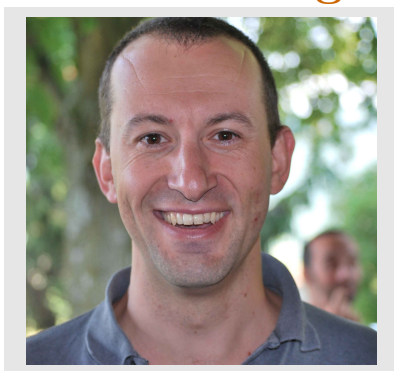

Stefano Gariglio is a Researcher in the Condensed Matter Physics Department at the University of Geneva. After his studies in Genoa (Italy), he obtained his Ph.D. in 2003 at the University of Geneva. His research interests are the physics of complex oxides, in particular, phenomena at interfaces and heterostructures, and their integration into functional devices.

\section{Jean-Marc Triscone}

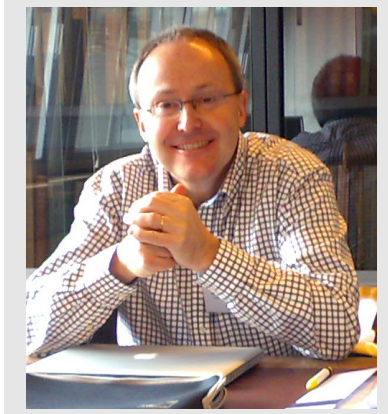

Jean-Marc Triscone is a Professor of Physics in the Condensed Matter Physics Department at the University of Geneva, leading a group working on nanoscale studies of ferroelectrics, electric field effects in correlated oxide systems, and oxide interface physics. He obtained his Ph.D. in 1987 with Professor Øystein Fischer. In 1990-1991 and in 1995 he was a visiting scientist at Stanford University. Since 1996, he has been a full Professor in Geneva. He has been a Fellow of the American Physical Society since 2006. His recent activities have focused on oxide interface physics, with the discovery of superconductivity at the $\mathrm{LaAlO}_{3} / \mathrm{SrTiO}_{3}$ interface.

\section{Andrea Caviglia}

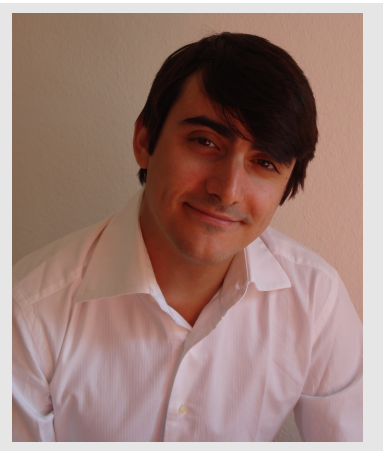

Andrea Caviglia is a postdoctoral researcher at the Max Planck Research Group for Structural Dynamics, Center for Free-Electron Laser Science, at the University of Hamburg. He received his Ph.D. from the University of Geneva. He is active in experimental condensed matter physics. His research interests include strongly correlated electrons and low-dimensional systems investigated by means of transport and ultrafast pump and probe spectroscopy. 